\title{
Rotational spectroscopy of imidazole: improved rest frequencies for astrophysical searches ${ }^{\star}$
}

\author{
B. M. Giuliano ${ }^{1}$, L. Bizzocchi ${ }^{1}$, A. Pietropolli Charmet $^{2}$, B. E. Arenas ${ }^{3}$, A. L. Steber $^{3,4}$, M. Schnell ${ }^{3,4}$, P. Caselli ${ }^{1}$,
} B. J. Harris ${ }^{5}$, B. H. Pate ${ }^{5}$, J.-C. Guillemin ${ }^{6}$, and A. Belloche ${ }^{7}$

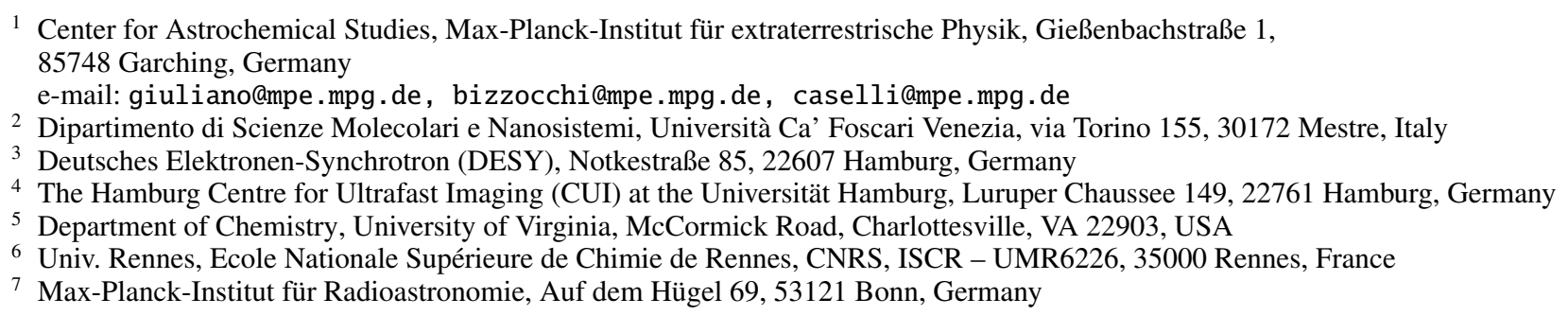

Received 25 April 2019 / Accepted 25 June 2019

\begin{abstract}
Context. Organic ring compounds play a key role in terrestrial biochemistry, and they were also most likely pivotal ingredients in Earth's prebiotic chemistry. The five-membered ring imidazole, $c-\mathrm{C}_{3} \mathrm{~N}_{2} \mathrm{H}_{4}$, is a substructure of fundamental biological molecules such as the purine nucleobases and the amino acid histidine. An unsuccessful search for imidazole in a sample of cold-core clouds and massive star-forming regions was performed almost 40 years ago. At that time, the spectroscopic knowledge of this species was scarce: the existing laboratory study was limited to the centimetre-wave region, and the precision of the rest frequencies in the millimetre regime was not adequate.

Aims. The goal of the present work is to perform a comprehensive investigation of the rotational spectrum of imidazole in its ground vibrational state from the microwave region to the $1 \mathrm{~mm}$ wavelength regime.

Methods. The rotational spectrum of imidazole was recorded in selected frequency regions from 2 to $295 \mathrm{GHz}$. These intervals were covered using various broadband spectrometers developed at DESY (Hamburg) and at the University of Virginia. High-level ab initio calculations were performed to obtain reliable estimates of the quartic and sextic centrifugal distortion constants. We used the EMoCA imaging spectral line survey to search for imidazole towards the hot molecular core Sgr B2(N2).

Results. About 700 rotational transitions spanning a $J$ interval from 0 to 59 and $K_{c}$ interval from 0 to 30 were analysed using the Watson $S$-reduced Hamiltonian. These new data allowed the determination of a much extended set of spectroscopic parameters for imidazole in its vibrational ground state. The improved spectral data allow us to set an upper limit to the column density of imidazole in Sgr B2(N2). Its non-detection implies that it is at least 3400 times less abundant than ethyl cyanide in this source.

Conclusions. With the new set of spectroscopic constants, it has been possible to compute reliable rest frequencies at millimetre wavelengths. We suggest a search for imidazole towards TMC-1, where the aromatic molecule benzonitrile was recently detected.
\end{abstract}

Key words. molecular data - methods: laboratory: molecular - techniques: spectroscopic - radio lines: ISM

\section{Introduction}

Heterocyclic aromatic compounds are a class of molecules that is characterised by a carbon ring structure in which one or more atoms are substituted by heteroatoms such as N, O, or S. Among these, $\mathrm{N}$-heterocycles are of great biological significance as they form the backbone of nucleobases, that is, subunits of DNA and RNA polymers, which carry the genetic information of living systems. Based on their structure, the five nucleobases can be considered as derivatives of pyrimidine $\left(c-\mathrm{C}_{4} \mathrm{H}_{4} \mathrm{~N}_{2}\right)$ or purine $\left(c-\mathrm{C}_{5} \mathrm{H}_{4} \mathrm{~N}_{4}\right)$. The latter is a two-ring aromatic compound obtained by the fusion of two structural moieties: the sixmembered pyrimidine $\left(c-\mathrm{C}_{4} \mathrm{H}_{4} \mathrm{~N}_{2}\right)$ and the five-membered

\footnotetext{
$\star$ Measured transition frequencies and catalogue of rest frequencies are only available at the CDS via anonymous ftp to cdsarc.u-strasbg.fr $(130.79 .128 .5)$ or via http://cdsarc. u-strasbg.fr/viz-bin/qcat?]/A+A/628/A53
}

imidazole $\left(c-\mathrm{C}_{3} \mathrm{H}_{4} \mathrm{~N}_{2}\right)$. Imidazole is also a structural part of other biologically relevant molecules, such as the amino acid histidine and the chemical mediator and neurotransmitter histamine.

Many N-heterocycles have been found in meteoritic materials (see, e.g. Botta \& Bada 2002; Martins 2018, and references therein), implying that these compounds can be produced under interstellar conditions. For example, gas-phase acetylene polymerisation in the presence of $\mathrm{HCN}$ is thought to generate nitrogen that contains rings up to large polycyclic aromatic structures (Frenklach \& Feigelson 1989; Ricca et al. 2001; Hamid et al. 2014). Moreover, grain-mantle reactions of $\mathrm{NH}_{3}$ in $\mathrm{H}_{2} \mathrm{O}$ ices doped with small quantities of polycyclic aromatic hydrocarbons produce traces of $\mathrm{N}$ - and $\mathrm{O}$-containing heterocycles and nucleobases under UV irradiation (Nuevo et al. 2014; Materese et al. 2015; Bera et al. 2017).

To date, relatively few organic ring molecules have been unambiguously identified in the interstellar medium (ISM). Among 
those detected are cyclopropenylidene $\left(\mathrm{c}-\mathrm{C}_{3} \mathrm{H}_{2}\right.$, Thaddeus et al. 1985), oxirane (c- $\mathrm{C}_{2} \mathrm{H}_{4} \mathrm{O}$, Dickens et al. 1997), cyclopropenone (c- $\mathrm{C}_{3} \mathrm{H}_{2} \mathrm{O}$, Hollis et al. 2006), benzene (c- $\mathrm{C}_{6} \mathrm{H}_{6}$, Cernicharo et al. 2001), and benzonitrile (c- $\mathrm{C}_{6} \mathrm{H}_{5}-\mathrm{CN}$, McGuire et al. 2018).

With regard to imidazole, one unsuccessful search was performed by Irvine et al. (1981), who targeted a small sample of sources including massive star-forming regions, dark clouds, and one carbon star. In all cases, only upper limits for the column density of this N-heterocycle could be obtained. The study was conducted using the Onsala $20 \mathrm{~m}$ telescope tuned in the centimetre domain to observe the $J_{K_{a} K_{c}}=313-212$ and the $J_{K_{a} K_{c}}=$ $3_{03}-2_{02}$ ground-state imidazole transitions, located at 33397.5 and $33416.2 \mathrm{MHz}$, respectively. However, spectral computations show that even at $T_{\text {exc }}=20 \mathrm{~K}$, the rotational features of imidazole already peak well into the millimetre regime $(\approx 110 \mathrm{GHz})$, thus making this region more promising for a successful astronomical search. Furthermore, during the past few decades, the performance of millimetre telescopes have witnessed an impressive improvement in terms of sensitivity, spectral coverage, and resolution, considerably increasing the chance of new molecular detections.

The current knowledge of the rotational spectrum of imidazole is inadequate for the purpose of present-day astrochemistry. Only two studies are present in the literature: Blackman et al. (1976) recorded a few transitions of the parent and the N-deutero isotopic variant in the $16-33 \mathrm{GHz}$ frequency interval using a Stark-modulation waveguide-based centimetre-wave spectrometer. A few years later, Christen et al. (1982) extended the study to 11 isotopologues and obtained experimental evaluations of the molecular structure, of the electric dipole moments, and an improved analysis of the nuclear quadrupole coupling that is generated by the nitrogen atoms. Despite the considerable amount of data presented in the latter paper, only limited centrifugal distortion constants were derived because of the small frequency coverage $(v<40 \mathrm{GHz})$ and the use of an outdated pre-Watson Hamiltonian (Watson 1977) to describe the rotational energy.

In this paper, we present a comprehensive investigation of the rotational spectrum of imidazole in its vibrational ground state. The use of advanced broadband spectrometers allowed us to record lines from the centimetre region $(\sim 2 \mathrm{GHz})$ to the submillimetre regime $(\sim 300 \mathrm{GHz})$, thus obtaining information on the quadrupole coupling of the two nitrogen nuclei, and an accurate analysis of the effects of the centrifugal distortion effects at high values of $J$ and $K_{c}$. In Sect. 2 we describe the experimental procedure and in Sect. 3 the data analysis. In Sect. 4 we provide a short account of the theoretical calculations performed to support the data analysis. In Sect. 5 we discuss the spectroscopic results and present an upper limit for imidazole in the ISM obtained from millimetre observations in Sgr B2(N2). We draw our conclusions in Sect. 6.

\section{Experiments}

The rotational spectrum of imidazole was recorded across the $2-295 \mathrm{GHz}$ frequency range employing several broadband chirped-pulse Fourier transform rotational spectrometers located at the Deutsches Elektronen-Synchroton (DESY; Hamburg) and at the University of Virginia. The Hamburg COMPACT spectrometer was used to record the spectrum in the 2-8 and $12-15.5 \mathrm{GHz}$ regions. For both measurements, the solid imidazole sample was loaded into a heatable reservoir nozzle, which was heated to $90^{\circ} \mathrm{C}$. The vapour was then supersonically expanded into the vacuum chamber with 3 bar of neon as the backing gas. The resulting rotational temperature of the sample is $T_{\text {rot }} \approx 1 \mathrm{~K}$. The electronic setup for the $2-8 \mathrm{GHz}$ measurement

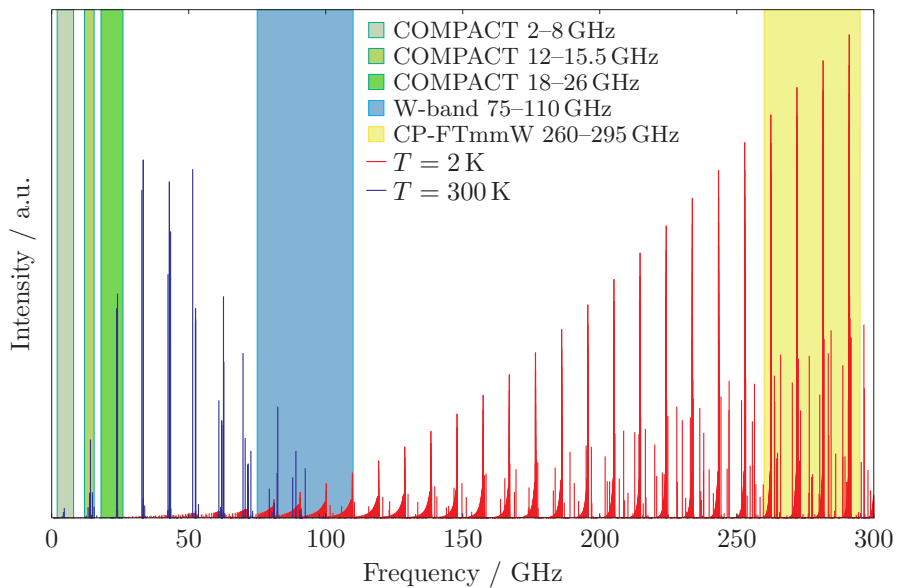

Fig. 1. Frequency intervals (shaded regions) covered by the measurements performed at DESY (COMPACT and $W$ band) and at the University of Virginia (CP-FTmmW). Two calculations of the rotational spectrum of imidazole at $2 \mathrm{~K}$ (blue sticks) and $300 \mathrm{~K}$ (red sticks) are shown overlapped to highlight the spectral sampling.

has previously been described elsewhere (Schmitz et al. 2012; Peréz et al. 2016), but a short explanation follows. Briefly, a train of eight successive chirped pulses is output by an arbitrary waveform generator (AWG) and amplified before being transmitted into the chamber. A $300 \mathrm{~W}$ travelling wave tube (TWT) amplifier is used to amplify the pulses. The relaxation of the excited molecules is then recorded in the form of a free induction decay (FID), which for this experiment was $40 \mu \mathrm{s}$. The final frequency-domain spectrum is obtained by fast Fourier transforming the one million co-added time-domain FIDs. The resulting frequency resolution of the spectrometer is $\sim 25 \mathrm{kHz}$. For the measurement between 12 and $15.5 \mathrm{GHz}$, the instrument operates in much the same way, except that a solid-state amplifier (Mercury Systems 6-18 GHz Solid State Amplifier) was used to amplify the chirped pulses instead of a TWT, and the FIDs are recorded for $20 \mu \mathrm{s}$.

For the measurements over $18-26 \mathrm{GHz}$, a newly built chirped-pulse Fourier transform microwave spectrometer in Hamburg was used. The same heatable reservoir nozzle was employed as in the COMPACT, and the sample was heated to $90^{\circ} \mathrm{C}$, and supersonically expanded into the chamber with 3 bar of neon. More details about the electronic setup will be provided in a later publication.

Unlike the previous experiments, the millimetre-wave $(\mathrm{mmW})$ measurements were performed at room temperature. For those taken in the 75-110 GHz range, a segmented chirped-pulse Fourier transform mmW (CP-FTmmW) spectrometer purchased from BrightSpec, Inc., was used. To maintain consistency, the same heatable reservoir nozzle used in the low-frequency measurements was employed to introduce the sample into the vacuum chamber. The sample was heated to $90^{\circ} \mathrm{C}$, and the resulting vapour was pulsed into the chamber, without the help of a backing gas, to maintain a constant pressure of $2 \mu$ bar in a slow-flow cell configuration. A full description of the experimental setup is given in Arenas et al. (2017), but a few key details are given here for reference. An AWG creates a series of excitation pulses (a pulse train) in the $2-5 \mathrm{GHz}$ range, which are then frequency up-converted and passed through an active multiplier chain (AMC) to produce mmW radiation. This is coupled into the vacuum chamber, where it excites the room-temperature molecules, before the FID of the relaxation is digitised and 
Fourier transformed into the frequency domain. In a segmented measurement, the spectrum across the entire bandwidth is constructed by collecting and adding together smaller segments of the rotational spectrum. In this case, the instrument's high dynamic range (HDR) mode was employed (Arenas et al. 2017), wherein the whole spectrum consists of $30 \mathrm{MHz}$ bandwidth segments. The total experiment time was approximately $100 \mathrm{~min}-$ utes for the one million FIDs to be recorded. The excitation pulses used here were $500 \mathrm{~ns}$ in duration, and each FID was recorded for $4 \mu \mathrm{s}$. The frequency accuracy of the spectrometer is $\sim 30 \mathrm{kHz}$, and line widths are about $550 \mathrm{kHz}$. The sample cell consists of a custom stainless steel chamber, with a path length of $72 \mathrm{~cm}$. For the COMPACT, the $18-26 \mathrm{GHz}$, and $\mathrm{mmW}$ measurements, a commercial sample of imidazole purchased from Sigma-Aldrich was used without further purification.

The higher frequency, room-temperature measurement from 260-295 GHz was performed at the University of Virginia. The CP-FTmmW spectrometer has been described previously (Steber et al. 2012; Neill et al. 2013; Harris 2014), and its operation is similar to that of the $75-110 \mathrm{GHz}$ spectrometer, with a few key differences. The AWG outputs a pulse train from $2-4 \mathrm{GHz}$, which is then frequency up-converted and passed through an AMC to reach 260-295 GHz. The HDR mode was also used in this experiment, and each $720 \mathrm{MHz}$ was addressed by thirty $24 \mathrm{MHz}$ chirps. The entire spectrometer band is addressed with fifty $720 \mathrm{MHz}$ segments. The pulse duration was $100 \mathrm{~ns}$, and the FID was collected for $1900 \mathrm{~ns}$. A three-minute 10000 shot HDR (Arenas et al. 2017) spectrum was acquired. The spectrometer sample cell is a $65 \mathrm{~cm}$ steel tube approximately $3.8 \mathrm{~cm}$ in diameter and one liter in volume. Polytetrafluoroethylene focal lenses act as windows to assist in beam collimation. In this experiment, purified imidazole crystals were used. The sample was placed into a sealed steel container equipped with a transfer valve. After removing the air, the sample was allowed to sublime and equilibrate at room temperature for $30 \mathrm{~min}$. Then, the transfer valve was opened slightly to set up a slow, steady flow at approximately $7 \mu$ bar. The flow cell was set up so as to minimize solid condensate in the transfer lines. A summary plot showing the spectral regions covered by the various set of measurements performed at DESY Hamburg and at the University of Virginia is presented in Fig. 1. They are indicated by shaded regions that overlap on two different stick spectra of imidazole computed at $2 \mathrm{~K}$ and $300 \mathrm{~K}$.

\section{Analysis}

Imidazole is a five-membered ring molecule containing two nitrogen atoms; its structure, the principal axes, and a conventional numbering of the skeleton atoms are depicted in Fig. 2. The delocalisation of six electrons (four double-bond $\pi$ and two of the $\mathrm{N}$ lone pair) provides improved stability of the ring skeleton (aromaticity) and constrains the molecule to a planar structure. From the spectroscopic point of view, imidazole is an oblate-type asymmetric top $(\kappa=0.8581)$ with dipole moment components of $\mu_{a}=3.603 \mathrm{D}$ and $\mu_{b}=0.680 \mathrm{D}$ (Christen et al. $1982)$. In the $\mathrm{mmW}$ region, the rotational spectrum is characterised by a sequence of dominant features spaced by $(A+B) / 2 \sim$ $9.5 \mathrm{GHz}$, which are composed of an $a$-type $Q$ transition ladder forming a band head overlapped by a compact series of strong $a$-type $R$ lines.

The $b$-type spectrum is much weaker, but it features a very similar pattern. This resemblance arises because for $K_{c} \gtrsim 8$, the levels $J_{J-K_{c}, K_{c}}$ and $J_{J-K_{c}+1, K_{c}}$ (asymmetry doublets) are almost degenerate, and thus the pairs of transitions ${ }^{a} Q_{+2,-1} /{ }^{b} Q_{+1,-1}$ and

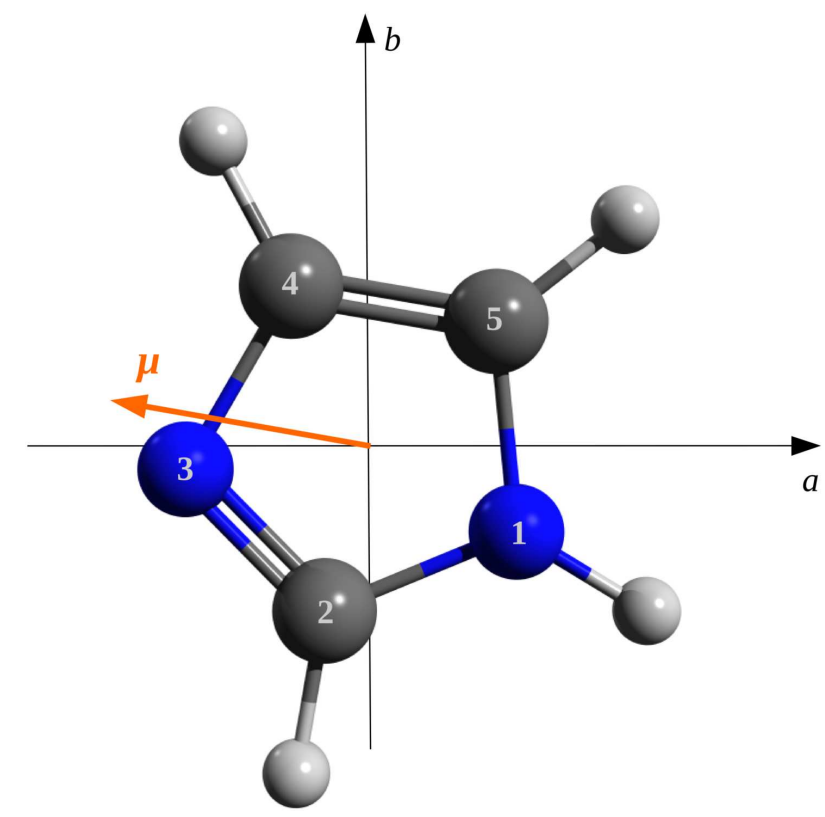

Fig. 2. Molecular structure and principal inertial axes of imidazole. The $c$ axis is perpendicular to the $a b$ molecular plane. The orange arrow indicates the direction of the electric dipole moment $\boldsymbol{\mu}$ and points towards the displacement of the notional negative charge.

${ }^{a} R_{0,+1} /{ }^{b} R_{+1,+1}$ fall essentially at the same frequency ${ }^{1}$. This is well illustrated in Fig. 3, which shows an excerpt of the imidazole spectrum recorded in the $3 \mathrm{~mm}$ spectral region.

The two nitrogen atoms (numbered as atoms 1 and 3 in Fig. 2, hereafter $\mathrm{N}^{(1)}$ and $\mathrm{N}^{(3)}$ ) generate the hyperfine structure (HFS) of the rotational transitions through the quadrupole coupling between the electric field gradient, averaged over the molecular rotation, and the $I=1$ spin of the ${ }^{14} \mathrm{~N}$ nuclei. This HFS is rather complex, and for low- $J$ transitions, it consists of a crowded pattern of components spread over a frequency interval as large as $4.5 \mathrm{MHz}$. Figure 4 presents two illustrative cases: the upper panel shows the crowded pattern of the $a$-type $1_{1,0}-1_{1,1}$ transition, which consists of 36 hyperfine components. On the opposite extreme, the lower panel displays the fundamental $b$ type line $1_{1,1}-0_{0,0}$, characterised by a loose HFS composed of only seven features.

Essentially, all the transitions recorded at frequencies below $26 \mathrm{GHz}$ show a resolvable HFS. They are summarised in Table 1, which also reports their hypothetically unsplit frequency and the number of assigned hyperfine components. When possible, a single hyperfine transition has been assigned to a given resolved feature detected in the rotational spectrum. Measurements corresponding to tight line blends have instead been assigned to the subset of components that make up the dominant intensity contribution. In these cases, the intensity-averaged frequency is compared with the experimental datum in the least-squares fit. Loose blends of unresolved components, resulting in very broad or distorted line profiles, have not been used in the analysis.

The hyperfine energies were computed adopting the standard vector coupling scheme between the rotational angular

1 The symbol ${ }^{x} M_{\delta K_{a}, \delta K_{c}}$ is used to label in a compact form the transition type for an asymmetric rotor: $x$ indicates the dipole moment component involved, $M=P, Q, R$ is the symbol for the transitions with $\Delta J=-1,0,+1$, respectively, and $\delta K_{a}$ and $\delta K_{c}$ refer to the (signed) change of the $K_{a}$ and $K_{c}$ pseudo-angular quantum numbers (Gordy \& Cook 1984). 


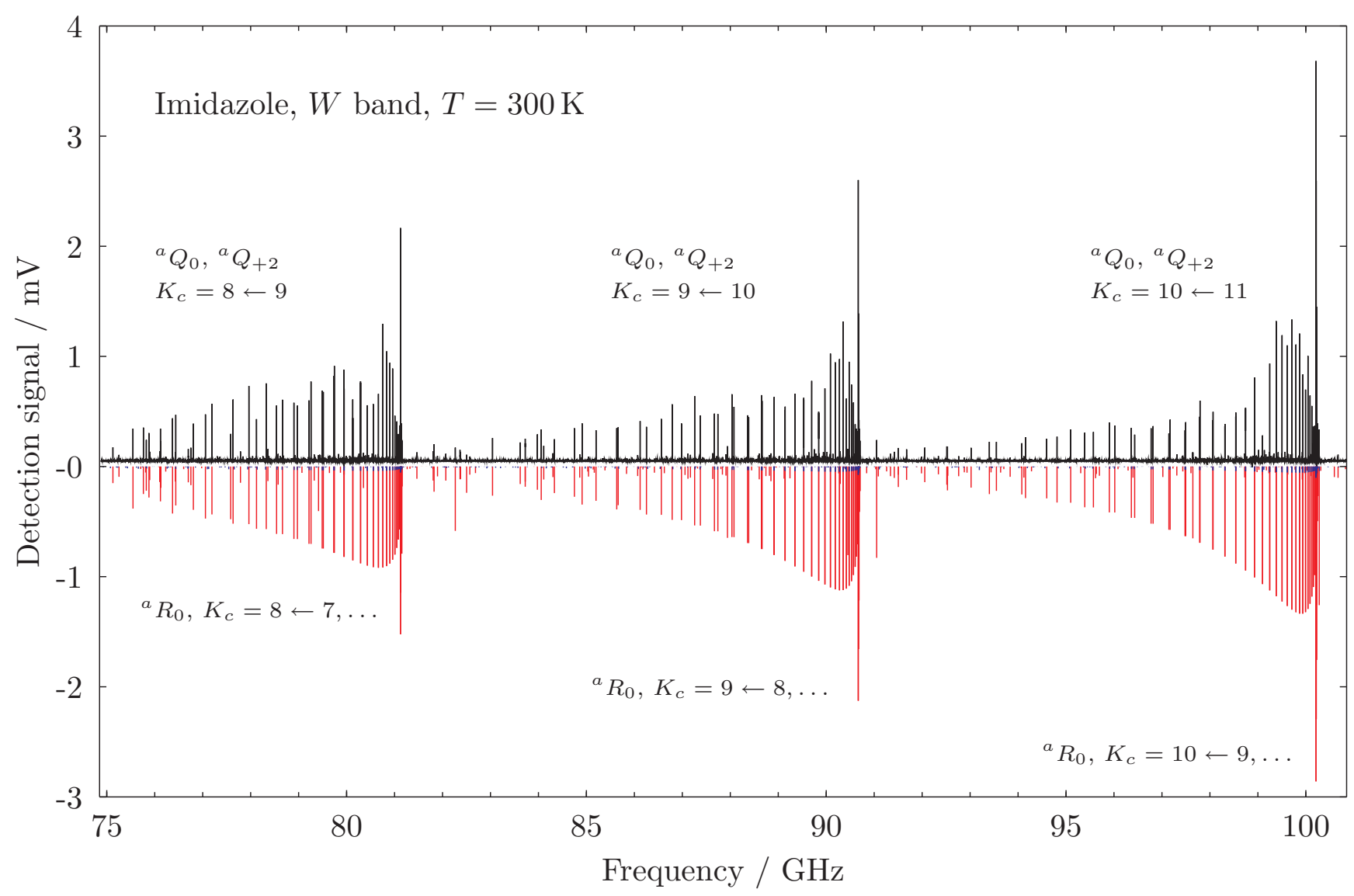

Fig. 3. Recording (black trace) of the imidazole rotational spectrum in the $3 \mathrm{~mm}$ region taken with the $W$-band segmented chirped-pulse spectrometer at DESY. The red histogram (inverted) indicates the predicted positions of the $a$-type lines computed using the spectroscopic parameters of Table 2 . The $b$-type transitions (blue trace) are very weak and barely visible in the used $y$-axis scale.

momentum $J$ and the two nitrogen spins $I_{N(1)}$ and $I_{N(3)}$ :

$\boldsymbol{J}+\boldsymbol{I}_{\mathrm{N}(1)}=\boldsymbol{F}_{\mathbf{1}}, \quad \boldsymbol{F}_{\mathbf{1}}+\boldsymbol{I}_{\mathrm{N}(3)}=\boldsymbol{F}$.

The total Hamiltonian is thus expressed as the sum of a purely rotational part and a hyperfine contribution:

$\hat{H}=\hat{H}_{\text {rot }}+\hat{H}_{\mathrm{HFS}}$.

Any hyperfine level is thus labelled with the quantum numbers $J_{K_{a}, K_{c}}, F_{1}, F$. The rotational Hamiltonian $\hat{H}_{\text {rot }}$ is the $S$-reduced Watson-type Hamiltonian in its $\mathrm{III}^{l}$ representation (suited for an oblate-type asymmetric rotor, Watson 1977) and includes centrifugal distortion corrections up to the octic terms. The hyperfine-structure Hamiltonian $\hat{H}_{\mathrm{HFS}}$ accounts for the nuclear quadrupole interactions of $\mathrm{N}^{(1)}$ and $\mathrm{N}^{(3)}$, each expressed by the traceless tensor $\chi^{(i)}$ with determinable coefficients $\chi_{c c}^{(i)}$ and $\chi_{b b}^{(i)}-\chi_{a a}^{(i)}$. Other hyperfine effects, such as spin-rotation and spinspin coupling, are very weak and do not produce any detectable effect in the recorded spectra.

The lines measured in the $W$ band $(75-110 \mathrm{GHz})$ and in the sub-mm regime $(260-295 \mathrm{GHz})$ generally do not show a detectable HFS of the rotational transitions because HFS decreases with increasing rotational quantum number. This subset constitutes the bulk of our experimental data set. For these data, the contribution due to the nuclear quadrupole coupling were neglected, and the experimentally measured frequencies were assigned to the corresponding pure rotational transitions.

In total, our data set comprises 698 transition frequencies. Different statistical weights $w=1 / \sigma^{2}$ were given to the various subsets of data in order to take the slightly different measurement precision $(\sigma)$ into account. A $\sigma=15 \mathrm{kHz}$ was given to the lines recorded with the COMPACT spectrometer, which used a jetcooled sample characterised by narrow line-widths. For the $W$ band and sub-mm measurements performed with static samples at room temperature, $\sigma=30 \mathrm{kHz}$ was used. The complete list of the measured transition frequencies is provided as electronic supplementary information.

\section{Theoretical calculations}

A high-level ab initio calculation of the molecular structure of imidazole has been reported previously (Császár et al. 2015). Here, we have performed a new computation aimed at obtaining a comprehensive set of theoretical estimates for its spectroscopic parameters. We adopted the coupled-cluster (CC) method considering single and double excitations augmented by a perturbative treatment for estimating the effects of triple excitations, that is, CCSD(T) (Raghavachari et al. 1989). This approach is today recognised as the gold standard for computational chemistry (see, e.g. Ramabhadran \& Raghavachari 2013; Puzzarini et al. 2014). The most relevant bias sources present in CCSD(T) calculations, mainly related to basis set and wave-function truncation effects, can be effectively accounted for using composite methods.

In the following, we briefly summarise the main steps of the computation procedure; a detailed account is given in Gambi et al. (2019). The complete basis set limit at the HF-SCF level of theory was derived using the extrapolation formula of 


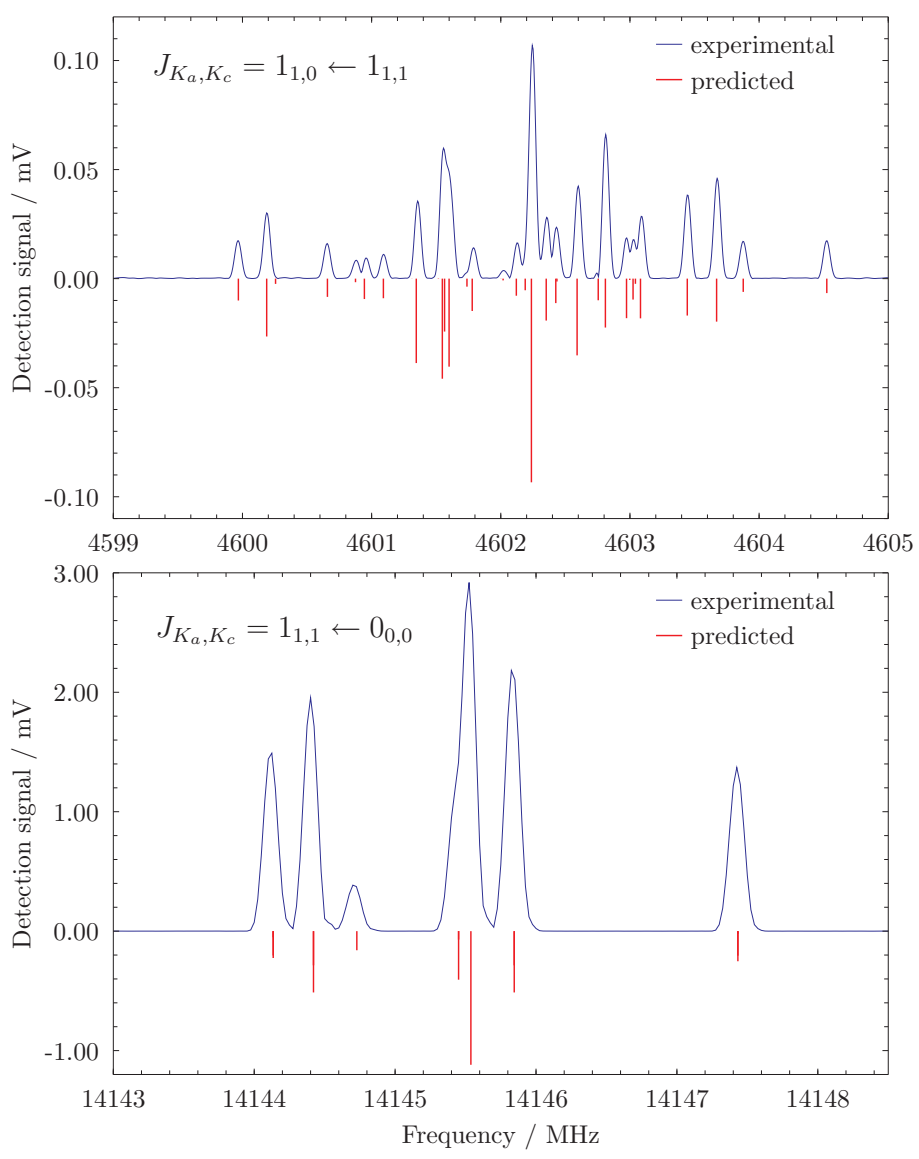

Fig. 4. Recordings (blue trace) of the $1_{1,0}-1_{1,1}$ (upper panel) and $1_{1,1}-0_{0,0}$ (lower panel) rotational transitions of imidazole taken with the COMPACT chirped-pulse spectrometer at DESY. The red histogram (inverted) indicates the predicted position of the hyperfine components computed using the spectroscopic parameters of Table 2.

Halkier et al. (1999) and using the correlation-consistent polarised basis sets cc-pVnZ ( $n=\mathrm{T}, \mathrm{Q}, 5)$ (Dunning 1989; Woon $\&$ Dunning 1995). For the correlation contribution, we used the two-parameter correction of Helgaker et al. (1997) by computing the corresponding terms at the $\operatorname{CCSD}(\mathrm{T})$ level of theory in the frozen-core (fc) approximation and employing the cc-pVTZ and cc-pVQZ basis sets. We computed the core-valence electron correlation contribution as the difference between the all-electron and frozen-core calculations carried out at the $\operatorname{CCSD}(\mathrm{T})$ level in conjunction with the cc-pCVTZ basis set (Woon \& Dunning 1995). In the end, from the structural parameters thus extrapolated, the corresponding equilibrium rotational constants were derived.

Given the good results yielded by composite schemes for also computing the properties related to the quadratic force field (see, e.g. Barone et al. 2015; Pietropolli Charmet et al. 2017a), we extrapolated the best estimate of the quartic centrifugal distortion constants by applying the same procedure followed for the structural parameters to the harmonic data calculated at the corresponding optimised geometries. To compute the vibrational corrections to equilibrium rotational constants and the data needed to evaluate the sextic centrifugal distortion constants, we employed the cubic force field obtained at the CCSD/cc-pVTZ level of theory, given the recognised good performances with respect to the more time-consuming CCSD(T) method (Pietropolli Charmet et al. 2017b; Senent et al. 2014).
Table 1. Recorded transitions that show a resolvable HFS.

\begin{tabular}{lccc}
\hline \hline Line & Type ${ }^{(a)}$ & Unsplit freq. (MHz) & No. of HFS lines \\
\hline $2_{0,2}-2_{2,1}$ & ${ }^{a} Q_{0,-1}$ & 4270.089 & 18 \\
$1_{1,0}-1_{1,1}$ & ${ }^{a} Q_{0,-1}$ & 4602.069 & 22 \\
$1_{1,0}-1_{0,1}$ & ${ }^{b} Q_{+1,-1}$ & 4953.383 & 15 \\
$2_{2,0}-2_{1,1}$ & ${ }^{b} Q_{+1,-1}$ & 5324.031 & 7 \\
$2_{1,1}-2_{1,2}$ & ${ }^{a} Q_{0,-1}$ & 13806.172 & 12 \\
$1_{0,1}-0_{0,0}$ & ${ }^{a} Q_{0,+1}$ & 14145.903 & 10 \\
$2_{2,1}-2_{0,2}$ & ${ }^{a} Q_{+2,-1}$ & 14879.470 & 5 \\
$2_{1,2}-1_{1,1}$ & ${ }^{a} R_{0,+1}$ & 23689.714 & 7 \\
$3_{1,2}-3_{1,3}$ & ${ }^{a} Q_{0,-1}$ & 23803.902 & 4 \\
$3_{2,2}-3_{0,3}$ & ${ }^{a} Q_{2,-1}$ & 23900.953 & 7 \\
$2_{0,2}-1_{0,1}$ & ${ }^{a} R_{0,+1}$ & 24021.675 & 4 \\
$2_{1,2}-1_{0,1}$ & ${ }^{b} R_{+1,+1}$ & 24041.029 & 1 \\
\hline
\end{tabular}

Notes. ${ }^{(a)}$ See footnote at page 3 .

Geometry optimisations and harmonic force-field data were performed using the CFOUR suite of programs ${ }^{2}$ and its implementation of analytic second derivatives (Gauß \& Stanton 1997), while to calculate the sextic centrifugal distortion constants, the VPT2 expressions (Aliev \& Watson 1976, 1985; Watson 1977) were adopted using an appropriate suite of programs (Pietropolli Charmet \& Cornaton 2018). Nuclear quadrupole coupling constants for the nitrogen atoms were computed at the CCSD(T)/ccpCVTZ level of theory and correlating all the electrons using the same procedure outlined in previous studies (Cazzoli et al. 2011; Pietropolli Charmet et al. 2016).

\section{Results and discussion}

\subsection{Spectral data}

The spectroscopic parameters for imidazole resulting from the analysis of the full data set of 698 lines are reported in Table 2. Table 2 contains the results of two different fits, together with a compilation of theoretically computed spectroscopic constants derived as described in Sect. 4. Precise determinations of the rotational constants $A, B, C$ were obtained, whose uncertainties are reduced by a factor of $\sim 100$ compared to previous findings (Christen et al. 1982). A full set of quartic centrifugal distortion constants were obtained for the first time. In previous works, the Watson-reduced (Watson 1977) form of the rotational Hamiltonian was not used (Christen et al. 1982 only quoted three $\tau$ unreduced quartic coefficients). The precision of our quartic centrifugal distortion constants is high, with relative $1 \sigma$ errors ranging in the $10^{-3}-10^{-5}$ interval. We also achieved a fair to good determination of the sextic constants $H_{J K}(\sim 1 \%), H_{K J}(\sim 5 \%)$, and $h_{2}(\sim 7 \%)$ and of the octic correction parameter $L_{J K K}(<10 \%)$. Our new values of the quadrupole coupling constants compare very well with those reported previously (Blackman et al. 1976; Christen et al. 1982), but their precision is improved by factors of $6-10$. For $\mathrm{N}^{(1)}$ the anisotropy term $\chi_{b b}-\chi_{a a}$ is too small to be determined from the present set of data. This is in agreement

2 CFOUR, Coupled-Cluster techniques for Computational Chemistry, a quantum-chemical program package written by J. F. Stanton, J. Gauss, L. Cheng, M. E. Harding, D. A. Matthews, P. G. Szalay et al., and the integral packages MOLECULE (J. Almlöf and P. R. Taylor), PROPS (P. R. Taylor), ABACUS (T. Helgaker, H. J. Aa. Jensen, P. Jørgensen, and J. Olsen), and ECP routines by A. V. Mitin and C. van Wüllen. For the current version, see http://www. cfour.de. 
Table 2. Experimental and theoretical spectroscopic parameters of imidazole.

\begin{tabular}{lcccc}
\hline \hline Parameter & & Fit I $^{(a)}$ & Fit II $^{(a)}$ & ab initio $^{(b)}$ \\
\hline$A$ & $/ \mathrm{MHz}$ & $9725.30651(18)$ & $9725.30901(19)$ & 9731.0337 \\
$B$ & $/ \mathrm{MHz}$ & $9373.99203(17)$ & $9373.99319(18)$ & 9387.3626 \\
$C$ & $/ \mathrm{MHz}$ & $4771.91599(18)$ & $4771.91736(19)$ & 4776.4213 \\
$D_{J}$ & $/ \mathrm{kHz}$ & $3.27363(15)$ & $3.27806(15)$ & 3.239 \\
$D_{J K}$ & $/ \mathrm{kHz}$ & $-5.17552(17)$ & $-5.17584(17)$ & -5.106 \\
$D_{K}$ & $/ \mathrm{kHz}$ & $2.24932(14)$ & $2.24728(14)$ & 2.210 \\
$d_{1}$ & $/ \mathrm{kHz}$ & $0.019825(17)$ & $0.020145(17)$ & 0.0217 \\
$d_{2}$ & $/ \mathrm{kHz}$ & $0.016675(10)$ & $0.016700(10)$ & 0.0194 \\
$H_{J}$ & $/ \mathrm{mHz}$ & $1.367^{(c)}$ & $0.0^{(c)}$ & 1.367 \\
$H_{J K}$ & $/ \mathrm{mHz}$ & $-6.148(51)$ & $-6.111(53)$ & -5.542 \\
$H_{K J}$ & $/ \mathrm{mHz}$ & $10.15(19)$ & $9.02(19)$ & 6.989 \\
$H_{K}$ & $/ \mathrm{mHz}$ & $-2.813^{(c)}$ & $0.0^{(c)}$ & -2.813 \\
$h_{1}$ & $/ \mathrm{mHz}$ & $0.0459^{(c)}$ & $0.0^{(c)}$ & 0.0459 \\
$h_{2}$ & $/ \mathrm{mHz}$ & $-0.0757(51)$ & $-0.1191(52)$ & -0.0607 \\
$h_{3}$ & $/ \mathrm{mHz}$ & $-0.0147^{(c)}$ & $0.0^{(c)}$ & -0.0147 \\
$L_{J K}$ & $/ \mu \mathrm{Hz}$ & $-1.98(10)$ & $-1.28(10)$ & - \\
$\chi_{c c}^{(1)}$ & $/ \mathrm{MHz}$ & $-2.5592(62)$ & $-2.5599(63)$ & -2.666 \\
$\chi_{b b}^{(1)}-\chi_{a a}^{(1)}$ & $/ \mathrm{MHz}$ & $0.0^{(c)}$ & $0.0^{(c)}$ & -0.071 \\
$\chi_{c c}^{(3)}$ & $/ \mathrm{MHz}$ & $2.2293(66)$ & $2.2293(66)$ & 2.210 \\
$\chi_{b b}^{(3)}-\chi_{a a}^{(3)}$ & $/ \mathrm{MHz}$ & $5.727(12)$ & $5.727(12)$ & 5.740 \\
$N_{0 .}$ of lines & & & 6.76 & \\
$\sigma_{\mathrm{w}}$ & & 0.76 & 0.76 & \\
\hline & & & & \\
\hline
\end{tabular}

Notes. Numbers in parentheses are $1 \sigma$ statistical uncertainties in the units of the last quoted digit. ${ }^{(a)}$ See Sect. 5 for an explanation. ${ }^{(b)}$ Equilibrium constants from extrapolated best structure, zero-point vibrational corrections computed at fc-CCSD/cc-pVTZ. Quartic centrifugal distortion constants computed using a composite scheme. Sextic centrifugal distortion constants computed at fc-CCSD/cc-pVTZ. Nuclear quadrupole coupling constants computed at the ae-CCSD(T)/fc-pCVTZ level. See Sect. 4 for further explanation. ${ }^{(c)}$ Fixed.

with the results of Christen et al. (1982), who performed a quadrupole coupling analysis of several isotopic species of imidazole and obtained individual determinations of each diagonal element of the $\chi$ tensor. From their results, it holds that $\chi_{b b}^{(1)}-\chi_{a a}^{(1)}=0.030(24)$, a value that is essentially zero within the quoted uncertainty.

The comparison of the experimentally derived spectroscopic parameters with those obtained from the theoretical calculations (reported in the rightmost column of Table 2) shows an excellent agreement. The ground-state rotational constants are predicted within $10 \mathrm{MHz}$, which corresponds to a $0.1 \%$ maximum discrepancy. Experimental and ab initio values of the quartic centrifugal distortion constants also compare well (within 1-2\% for the diagonal $D_{J}, D_{J K}$, and $D_{K}, 10-20 \%$ for the small $d_{1}$ and $d_{2}$ ). Furthermore, for the three determined sextic constants, the sign and the order of magnitude have been correctly predicted by theory.

Fit I and fit II (Table 2) differ for the different choice of the assumed value of the sextic centrifugal distortion constants, which could not be adjusted in the least-squares analysis, that is, $H_{J}, H_{K}$, $h_{1}$, and $h_{3}$. In fit I, these parameters were held fixed at the corresponding theoretically computed values (column "ab initio"), while in fit II these constants were simply constrained to zero. As expected, the two sets of spectroscopic constants are slightly different, reflecting the existing correlations between the model parameters, for instance, $H_{J}$ with $C$ and $D_{K} ; H_{K}$ with $A, B, C$, and $D_{J}$. The comparison between spectral calculations based on fit I and fit II constants make it possible to derive a more reliable error bar (beyond simple error propagation laws) for any individual rest frequency. In particular, this provides a sound evaluation of the model error, that is, the arbitrary assumptions made for the
Hamiltonian coefficients that cannot be determined from the available experimental data set.

To quantify this effect, we selected a subset of strong spectral features with rest frequency $v_{\mathrm{rf}}<300 \mathrm{GHz}$, originated from levels with $E_{u} / k<250 K$ (where $k$ is the Boltzmann constant) and with integrated intensity down to the $10 \%$ of the maximum computed at $T_{\mathrm{ex}}=30 \mathrm{~K}$. For this subset of 1485 lines, which are the most important for astronomical purposes, we evaluated the quantity

$d=\frac{\left|v_{\mathrm{rf}}^{\mathrm{fit}}-v_{\mathrm{rf}}^{\mathrm{fit}}\right|}{\sigma}$,

which ranged in the 0.03-10.4 interval with a median value of 2.71 . The results indicate that quoting a $3 \sigma$ statistical uncertainty is essentially safe for $50 \%$ of the rest frequency set, but a significant fraction of the sample (especially lines with high $J, K_{c}$ values) might be affected by larger systematic errors. This is due to the assumptions made on the fixed sextic centrifugal distortion constants and possibly to some missing higher order parameters.

The electronic supplementary information contains a catalogue of rest frequencies for imidazole that were directly obtained with the SPCAT programme (Pickett 1991) without any further editing. This data listing exactly matches the file format of $\mathrm{CDMS}^{3}$ (Müller et al. 2005; Endres et al. 2016) and JPL ${ }^{4}$ (Pickett et al. 1998) databases, and can thus readily be implemented in some of the most frequently used astronomy analysis tools, such as CASSIS ${ }^{5}$ and MADCUBA (Rivilla et al. 2016).

\footnotetext{
3 https://cdms.astro.uni-koeln.de/cdms

4 http://spec.jpl.nasa.gov/

5 CASSIS is a software package for the analysis of astronomical spectra developed by IRAP-UPS/CNRS (http://cassis.irap.omp . eu).
} 
Table 3. Rotational, hyperfine, and vibrational partition functions of imidazole.

\begin{tabular}{lcrc}
\hline \hline$T / \mathrm{K}$ & \multicolumn{1}{c}{$Q_{\text {rot }}$} & \multicolumn{1}{c}{$Q_{\text {HFS }}$} & $Q_{\text {vib }}$ \\
\hline 3 & 43.092 & 387.83 & 1.0000 \\
5 & 91.766 & 825.90 & 1.0000 \\
10 & 257.57 & 2318.09 & 1.0000 \\
15 & 471.98 & 4247.77 & 1.0000 \\
25 & 1013.47 & 9121.30 & 1.0000 \\
50 & 2862.01 & 25758.2 & 1.0000 \\
100 & 8041.27 & 72371.5 & 1.0008 \\
150 & 14487.2 & 130385 & 1.0013 \\
225 & 25455.6 & 229101 & 1.1051 \\
300 & 37264.1 & 335377 & 1.3522 \\
\hline
\end{tabular}

The results of two separate computations are provided. The file imidazole. cat contains a compilation of pure rotational frequencies extending up to $600 \mathrm{GHz}$, and the file imidazole_ hfs.cat provides a list of hyperfine components limited to $50 \mathrm{GHz}$. Both catalogues were obtained from fit I results (see Table 2), and following the CDMS standard, the integrated intensity of each transition is computed at $300 \mathrm{~K}$. The quoted errors are three times the genuine statistical uncertainty $(3 \sigma)$ or the quantity $\left|v_{\mathrm{rf}}^{\text {fit I }}-v_{\mathrm{rf}}^{\mathrm{fit}}\right| \mathrm{II}$ used in Eq. (3), whichever is greater.

A listing of the rotational $\left(Q_{\text {rot }}\right)$, hyperfine $\left(Q_{\mathrm{HFS}}\right)$, and vibrational $\left(Q_{\mathrm{vib}}\right)$ partition functions of imidazole is provided in Table 3. They are computed for temperatures ranging in the 3-300 K interval. These values are computed by direct summation over the rotational levels whose energy position is accurately determined during the spectral analysis. The vibrational partition functions are obtained through direct summation on all energy levels (including combinations and overtones), which contribute to $Q_{\text {vib }}$ higher than $10^{-7}$. The vibrational energies were derived theoretically using a hybrid method that employs harmonic $\operatorname{CCSD}(\mathrm{T})$ calculations with complete basis set extrapolation, plus an MP2 estimate of the anharmonic contribution (Pietropolli Charmet et al., in prep.).

\subsection{Astrophysical implications}

We used the best-fit model of the rotational spectrum of imidazole obtained in this work to search for this molecule in the hot molecular core Sgr B2(N2). This hot core is located in the Sagittarius B2 molecular cloud, at a distance of $8.3 \mathrm{kpc}$ (Reid et al. 2014). We used the EMoCA imaging spectral line survey obtained with the Atacama Large Millimeter/submillimeter Array between $84.1 \mathrm{GHz}$ and $114.4 \mathrm{GHz}$ with a median angular resolution of $1.6^{\prime \prime}$. A detailed account of the observations and data reduction can be found in Belloche et al. (2016). We used Weeds (Maret et al. 2011) to generate a synthetic spectrum of imidazole under the approximation of local thermodynamic equilibrium (LTE) and searched for matching lines in the observed spectrum of Sgr B2(N2).

We did not find evidence for imidazole in the EMoCA spectrum of Sgr B2(N2). The most stringent constraints are imposed by the transitions around $100.21 \mathrm{GHz}$ (see Fig. 5). The line detected at $\sim 100.21 \mathrm{GHz}$ towards Sgr B2(N2) is a blend of transitions of $\mathrm{CH}_{3}^{18} \mathrm{OH},{ }^{13} \mathrm{CH}_{3} \mathrm{CH}_{2} \mathrm{OH}$, and $\mathrm{CH}_{3} \mathrm{C}(\mathrm{O}) \mathrm{CH}_{3}$. There is little room left for imidazole emission at this frequency. Assuming the same LTE parameters as for ethyl cyanide (a source size of $1.2^{\prime \prime}$, a temperature of $150 \mathrm{~K}$, a line width of $5 \mathrm{~km} \mathrm{~s}^{-1}$, and a source velocity of $73.2 \mathrm{~km} \mathrm{~s}^{-1}$ ), we obtain an upper limit

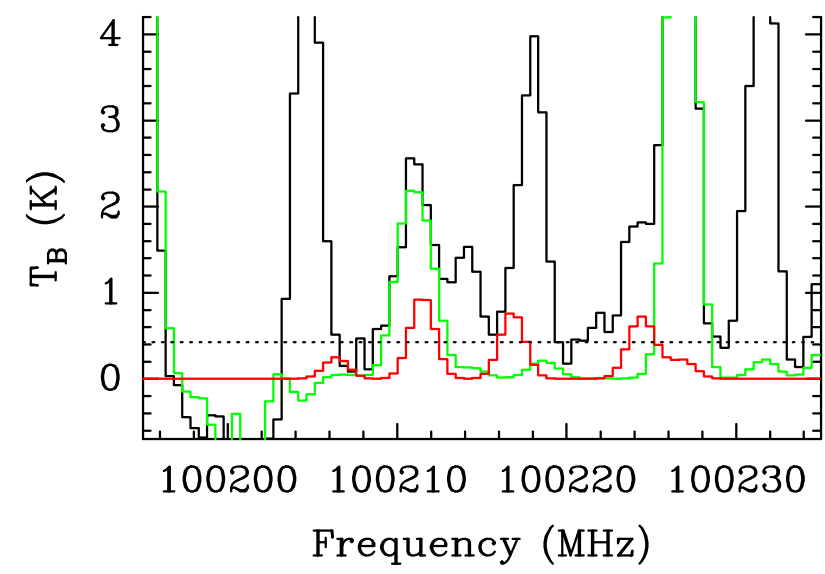

Fig. 5. Portion of the EMoCA survey around $102.2 \mathrm{GHz}$. The best-fit LTE synthetic spectrum that contains the contributions of all molecules identified so far towards Sgr B2(N2) is overlaid in green on the observed spectrum, which is shown in black. The synthetic spectrum of imidazole used to derived the upper limit on its column density is shown in red. The $y$-axis is labelled in brightness temperature units. The dotted line indicates the $3 \sigma$ noise level.

of $1.8 \times 10^{15} \mathrm{~cm}^{-2}$ for the column density of imidazole, which implies that this ring is at least 3400 and 230 times less abundant than ethyl cyanide and vinyl cyanide in this source, respectively

(Belloche et al. 2016). At a temperature of $150 \mathrm{~K}$, the vibrational contribution to the partition function of imidazole would increase the derived value of the column density upper limit by only $0.1 \%$ (see Table 3 ). McGuire et al. (2018) reported a column density of $4 \times 10^{11} \mathrm{~cm}^{-2}$ for benzonitrile $\left(c-\mathrm{C}_{6} \mathrm{H}_{5} \mathrm{CN}\right)$ in TMC-1, which is 15 times less abundant than vinyl cyanide in this source (Gratier et al. 2016). If imidazole and benzonitrile have similar abundances in Sgr B2(N2), then we may conclude that aromatic molecules are less abundant towards Sgr B2(N2) than towards TMC-1 relative to aliphatic compounds.

\section{Conclusions}

This paper presents a comprehensive laboratory study of the rotational spectrum of imidazole, extending the earlier microwaveband investigations into the millimetre-wave regime. The use of different broadband spectrometers allowed us to sample with continuity wide portions of the spectrum: at centimetre wavelength, the intervals $2-8 \mathrm{GHz}, 13-15.5 \mathrm{GHz}$, and $18-26 \mathrm{GHz}$ were covered, and in the mmW region, the full $\mathrm{W}$-band $(75-110 \mathrm{GHz})$ and the $260-295 \mathrm{GHz}$ range were studied. The low- $J$ lines recorded below $26 \mathrm{GHz}$ show complex hyperfine patterns due to the quadrupole coupling of the two ${ }^{14} \mathrm{~N}$ nuclei. For these transitions, 119 separate components were accurately measured. The final analysis comprises 698 transitions, which were fitted to the coefficients of the $S$-reduced rotational Hamiltonian, providing a very precise set of rotational and quartic centrifugal distortion constants. Three sextic and one octic parameters were also determined. The optimised values of the spectroscopic constants are in excellent agreement with the results of high-level theoretical calculations, which were performed to assist the present analysis and as a basis for future investigation focused on the imidazole isotopologues and its low-lying vibrationally excited states. This paper provides the first set of complete spectroscopic parameters of imidazole, thus allowing us to compute reliable rest frequencies in the $\mathrm{mmW}$ regime, a spectral region in which the features of this ring molecule reach their maximum intensity at typical ISM 
gas temperatures. Using these improved spectral predictions, a search for imidazole was attempted in the hot core Sgr B2(N2) based on the observations of the EMoCA spectral line survey. This ring molecule is not detected. We derive a column density upper limit of $1.8 \times 10^{15} \mathrm{~cm}^{-2}$, which implies that imidazole is at least 3400 times less abundant than ethyl cyanide in this source. Given that the cyclic molecule benzonitrile $\left(c-\mathrm{C}_{6} \mathrm{H}_{5} \mathrm{CN}\right)$ was recently detected in the molecular cloud TMC-1 (McGuire et al. 2018), the chances of detecting imidazole may be higher towards this source.

Acknowledgements. M. Schnell acknowledges the ERC Starting Grant ASTROROT (Grant Agreement Number 638027). B. E. Arenas acknowledges The International Max Planck Research School for Ultrafast Imaging and Structural Dynamics, IMPRS-UFAST. A. L. Steber is grateful for The Louise Johnson Fellowship of the Hamburg Centre for Ultrafast Imaging, CUI. B. J. Harris and B. H. Pate are supported by National Science Foundation. J.-C. Guillemin thanks the Centre National d'Etudes Spatiales (CNES) for financial support. A. Pietropolli Charmet gratefully acknowledges financial support by University Cà Foscari Venezia (ADiR funds) and the SCSCF ("Sistema per il Calcolo Scientifico di Cà Foscari”, a multiprocessor cluster system owned by Università Cà Foscari Venezia). This work has been in part supported by the Deutsche Forschungsgemeinschaft (DFG) through the collaborative research grant SFB 956 "Conditions and Impact of Star Formation", project area B3.

\section{References}

Aliev, M. R., \& Watson, J. K. G. 1976, J. Mol. Spectr., 61, 29

Aliev, M. R., \& Watson, J. K. G. 1985, in Molecular Spectroscopy: Modern Research, ed. K. N. Rao (New York: Academic Press), III, 1

Arenas, B. E., Gruet, S., Steber, A. L., Giuliano, B. M., \& Schnell, M. 2017 Phys. Chem. Chem. Phys., 19, 1751

Barone, V., Biczysko, M., \& Puzzarini, C. 2015, Acc. Chem. Res., 48, 1413

Belloche, A., Müller, H. S. P., Garrod, R. T., \& Menten, K. M. 2016, A\&A, 587, A91

Bera, P. P., Stein, T., Head-Gordon, M., \& Lee, T. J. 2017, Astrobiology, 17, 771

Blackman, G. L., Brown, R. D., Burden, F. R., \& Elsum, I. R. 1976, J. Mol. Spectr., 60, 63

Botta, O., \& Bada, J. L. 2002, Surveys Geophys., 23, 411

Cazzoli, G., Cludi, L., Puzzarini, C., et al. 2011, J. Phys. Chem. A, 115, 453

Cernicharo, J., Heras, A. M., Tielens, A. G. G. M., et al. 2001, ApJ, 546, L123

Christen, D., Griffith, J. H., \& Sheridan, J. 1982, Z. Naturforsch., 37, 1378

Császár, A., Demaison, J., \& Rudolph, H. D. 2015, J. Phys. Chem. A, 119, 1731

Dickens, J. E., Irvine, W. M., Ohishi, M., et al. 1997, ApJ, 489, 753

Dunning, Jr., T. H. 1989, J. Chem. Phys., 90, 1007

Endres, C. P., Schlemmer, S., Schilke, P., Stutzki, J., \& Müller, H. S. P. 2016, J. Mol. Spectr., 327, 95

Frenklach, M., \& Feigelson, E. D. 1989, ApJ, 341, 372

Gambi, A., Pietropolli Charmet, A., Stoppa, P., et al. 2019, Phys. Chem. Chem. Phys., 21, 3615
Gauß, J., \& Stanton, J. F. 1997, Chem. Phys. Lett., 276, 70

Gordy, W., \& Cook, R. L. 1984, Microwave molecular spectra (New York: Wiley)

Gratier, P., Majumdar, L., Ohishi, M., et al. 2016, ApJS, 225, 25

Halkier, A., Helgaker, T., Jørgensen, P., Klopper, W., \& Olsen, J. 1999, Chem. Phys. Lett., 302, 437

Hamid, A. M., El-Shall, M. S., Hilal, R., Elroby, S., \& Aziz, S. G. 2014, J. Chem. Phys., 141, 054305

Harris, B. J. 2014, PhD Thesis, University of Virginia, USA

Helgaker, T., Klopper, W., Koch, H., \& Noga, J. 1997, J. Chem. Phys., 106 , 9639

Hollis, J. M., Remijan, A. J., Jewell, P. R., \& Lovas, F. J. 2006, ApJ, 642, 933

Irvine, W. M., Ellder, J., Hjalmarson, A., et al. 1981, A\&A, 97, 192

Maret, S., Hily-Blant, P., Pety, J., Bardeau, S., \& Reynier, E. 2011, A\&A, 526, A47

Martins, Z. 2018, Life, 8, 28

Materese, C. K., Nuevo, M., \& Sandford, S. A. 2015, ApJ, 800, 116

McGuire, B. A., Burkhardt, A. M., Kalenskii, S., et al. 2018, Science, 359, 202

Müller, H. S. P., Schlöder, F., Stutzki, J., \& Winnewisser, G. 2005, J. Mol. Struct. 742,215

Neill, J. L., Harris, B. J., Steber, A. L., et al. 2013, Opt. Exp., 21, 19743

Nuevo, M., Materese, C. K., \& Sandford, S. A. 2014, ApJ, 793, 125

Peréz, C., Krin, A., Steber, A. L., et al. 2016, J. Phys. Chem. Letters, 7, 154

Pickett, H. M. 1991, J. Mol. Spectr., 148, 371

Pickett, H. M., Poynter, R. L., Cohen, E. A., et al. 1998, J. Quant. Spectr. Radiat. Transf., 60, 883

Pietropolli Charmet, A., \& Cornaton, Y. 2018, J. Mol. Struct., 1160, 455

Pietropolli Charmet, A., Stoppa, P., Tasinato, N., Giorgianni, S., \& Gambi, A. 2016, J. Phys. Chem. A, 120, 8369

Puzzarini, C., Biczysko, M., Bloino, J., \& Barone, V. 2014, ApJ, 785, 107

Pietropolli Charmet, A., Stoppa, P., Giorgianni, S., et al. 2017a, J. Phys. Chem. A, 121, 3305

Pietropolli Charmet, A., Stoppa, P., Tasinato, N., \& Giorgianni, S. 2017b, J. Mol. Spectr., 335, 117

Raghavachari, K., Trucks, G. W., Pople, J. A., \& Head-Gordon, M. 1989, Chem. Phys. Lett., 157, 479

Ramabhadran, R. O., \& Raghavachari, K. 2013, J. Chem. Theor. Comp., 9 3986

Reid, M. J., Menten, K. M., Brunthaler, A., et al. 2014, ApJ, 783, 130

Ricca, A., Bauschlicher, C. W., \& Bakes, E. L. O. 2001, Icarus, 154, 516

Rivilla, V. M., Fontani, F., Beltrán, M. T., et al. 2016, ApJ, 862, 161

Schmitz, D., Alvin Shubert, V., Betz, T., \& Schnell, M. 2012, J. Mol. Spectr., 280, 77

Senent, M. L., Puzzarini, C., Hochlaf, M., Domínguez-Gómez, R., \& Carvajal, M. 2014, J. Chem. Phys., 141, 104303

Steber, A. L., Harris, B. J., Neill, J. L., \& Pate, B. H. 2012, J. Mol. Spectr., 280, 3

Thaddeus, P., Vrtilek, J. M., \& Gottlieb, C. A. 1985, ApJ, 299, L63

Watson, J. K. G. 1977, in Vibrational Spectra and Structure, ed. J. Durig (Amsterdam: Elsevier), 6, 1

Watson, J. K. G. 1977, J. Mol. Spectr., 65, 123

Woon, D. E., \& Dunning, T. H. 1995, J. Chem. Phys., 103, 4572 